\title{
Deployment of Tactical Mobile Networks
}

\author{
Dalal Alshammari ${ }^{1} \mid$ Salman Al-Shehri ${ }^{1}$ \\ ${ }^{1}$ Swansea University, Swansea SA18EN, United Kingdom. \\ To Cite this Article \\ Dalal Alshammari and Salman Al-Shehri, "Deployment of Tactical Mobile Networks", International Journal for Modern \\ Trends in Science and Technology, Vol. 06, Issue 07, July 2020, pp.:27-29; https://doi.org/10.46501/IJMTST060704
}

\section{Article Info}

Received on 16-March-2020, Revised on 22-April-2020, Accepted on 02-May-2020, Published on 27-June-2020.

\section{ABSTRACT}

Tactical mobile networks are at the forefront of military communication systems during military missions and in digitalized battlefields, and they can be also used for disaster recovery and rescue missions. These networks serve diverse range of applications to users with different mobility patterns and operate under adverse radio propagation conditions. They are highly dynamic and heterogeneous and must provide minimum level of connectivity and coverage without any supporting infrastructure. This makes their design and deployment much more challenging with much stringent requirements than for other types of mobile communication networks. As the complexity of telecommunication systems has increased together with many intricate relationships among various stakeholders, the selection and usage of network metrics has become much more important. Network metrics are crucial to define key performance indicators (KPIs) and service quality levels whilst some metrics have been standardized. Common telecommunication metrics have been reviewed and then specialised for tactical networks to understand different trade-offs such as reliable coverage versus quality of service. The collected metrics were then sorted into several main categories with majority of these metrics being either related to evaluating the system performance or evaluating the user experience.

KEYWORDS: recovery, soccer players, muscle damage

Copyright (C) 2014-2020 International Journal for Modern Trends in Science and Technology

DOI: https://doi.org/10.46501/IJMTST060704

\section{INTRODUCTION}

Legacy cellular networks exploit a BTS/BTC (base stations and their controllers) architecture with high-speed backhaul links to offer the radio access networks (RANs) to wireless subscribers [1]. Recently, the $5 \mathrm{G}$ networks envision direct device-to-device ( $\mathrm{d} 2 \mathrm{~d}$ ) communications in addition to the traditional uplink (device-to-BTS) and downlink (BTS-to-device) connectivity [2]. There are also many applications of wireless networks where it is impractical to plan and deploy the supporting network infrastructure (i.e., RAN), so the network topology is established by enabling direct $\mathrm{d} 2 \mathrm{~d}$ communications [3]. The resulting ad-hoc or mesh topology of Mobile Ad hoc NETwork
(MANETs) and Vehicular Ad-hoc NETworks (VANETs) supports mobility of the network nodes [4].

MANETs are a collection of wireless nodes communicating with each other in the absence of any other infrastructure. Due to the availability of small and inexpensive wireless communicating devices, MANETs have attracted a lot of attention from the academia and industry in recent years. Moreover, the global competition is driving the telecommunications industry towards ubiquitous computing achieved "anytime, anywhere with/to anything" locally as well as globally to meet the customer expectations and satisfaction. MANETs can be used in various applications such as mobile 
classrooms, communications in battlefield scenarios, sensor networks and they are also used for personal area networks (PANs), internet of things (IoT) and disaster relief applications. Hence, with ongoing advances in wireless technologies, MANET will play a significant role in enabling many important applications and services. However, MANETs also pose many severe challenges in their deployment and operations because of their special characteristics such as multi-paths propagations and multi-hop packet delivery without access points leading to their dynamic topology. This represents other challenges with routing, quality of service (QoS), battery, bandwidth and security.

\section{Methodology}

Tactical MANETs (also known as mobile tactical networks) can be categorised as MANETs where the system are fully distributed and dynamic. In particular, these networks are specifically designed to extend the capabilities of the general MANETs. In 2 past decades, defence-sponsored research of military communications was the main drive behind numerous of today's communications technologies. In fact, conventional MANETs have been primarily designed to enhance tactical communications in a battlefield. In the early 1970s, the Defence Advanced Research Projects Agency (DARPA) designed the first generation of MANETs, called packet radio network (PRNeT). The primary aim of this network is to provide reliable coverage, especially for voice service [5]. Tactical MANETs (T-MANETs) then gradually evolved to focus on improving their range, capabilities and security, as Figure 1.1 shows.

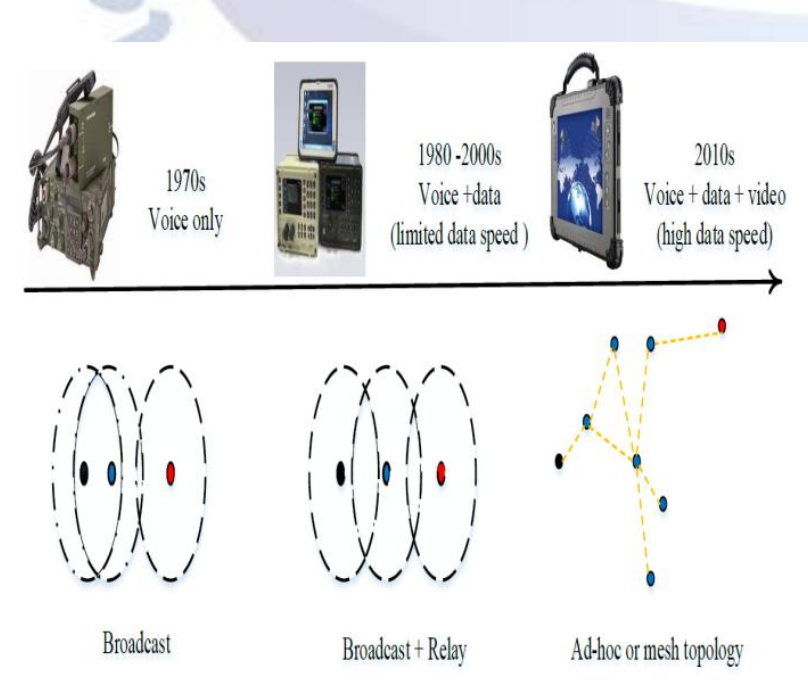

Figure 1.1. The evolution of tactical communications.
Military networks, especially tactical ones, differ significantly from those conventional MANET networks. For example, general ad-hoc networks assumed that all nodes have similar behaviours and capabilities [6], where tactical ad-hoc networks can range from small squads of soldiers to high mobility unmanned aerial vehicle (UAV) networks. Moreover, T-MANETs are exposed to external interference, jamming and link quality fluctuating. There is an ever-increasing need to understand the actual mobility behaviour and propagation environments. Accurate modelling of radio propagation environment can provide an accurate prediction of radio coverage in the operational area. Consequently, the requirements for military radios are much more stringent than those expected for commercial radios.

\section{RESULTS}

The key design focus of these networks is on robustness, survivability and mobility to offer communication services for different types of users and applications within challenging environments with difficult and hostile radio propagation conditions and with no supporting infrastructure available.

T-MANETs must be able to meet certain minimum requirements which are essential for their deployment and operation. In particular, T-MANETs must offer reliable communication networks which deliver some basic defined services. This is generally preferable to a less reliable network offering high-end services. T-MANETs are expected to support bandwidth-intensive, delay sensitive and real-time applications despite the limited available spectrum. T-MANETs must be scalable in order to accommodate varying numbers of tactical units and to seamlessly integrate with other battlefield networks. Achieving LOS connectivity for T-MANETs is vital. This is even more important when T-MANETs are used for tactical missions carried out in the mountainous areas and other hilly terrains. Other key considerations when deploying T-MANETS are transmission ranges, and transmission capacity issues to enable effective communications in the field. For some tactical missions, it is possible to exploit pre-planned movement trajectories of tactical units as well as having location information at each such unit in order to improve the network connectivity. Moreover, transceivers in T-MANETs must use the same interface for all types and different roles of 
their operations. For instance, tactical communications system must be able to support tactical missions in high-density urban areas as well as in widely dispersed environments.

T-MANETs are often designed and evaluated by the means of computer simulations. It is desirable to compare simulation results against field data to improve modelling and reach better predictive power of simulations. Unlike conventional MANETs, models of tactical networks must consider also behavior of entities including human aspects within the complex warfare scenarios. For example, operational phases of the mission such as defence and attack are affected by tactical formation of units and troops. These issues are not technical matters, but they may represent the greatest hurdles to be overcome. In addition, there are several abstract and intangible components affecting military communications such as the experiences, doctrines and different aspects of human behaviour [7].

\section{DISCUSSION}

T-MANETs have changed much over the years. Currently, tactical networks represent complex communication infrastructures providing heterogeneous connectivity in tactical missions and digitalised battlefields. Today's hardware components provide

sufficient computing and storage enabling to abstract many processes entirely in software. For instance, software-defined networks (SDN), network function virtualisation (NFV) and network analytics provide unprecedented flexibility in configuring communication services while optimising the utilisation of network resources. Consequently, the frequency agility and interoperability requirements, for example, can be resolved by SDR operated across a wide range of frequencies. Moreover, to reduce development times and costs for tactical networks, commercial off-the-shelf technologies (COTS) including 4G/5G networks can be adopted for use in tactical networks, which creates new opportunities as well as challenges. On the other hand, the future networks will be more autonomous, i.e., self-organising, self-healing, self-secure and self-optimising. The heterogeneous nature of the next generation tactical networks will require new metrics and management strategies. The traditionally used metrics must be updated to remain relevant since the underlying assumptions of their use have changed. For example, the battery lifetime of portable transceivers in tactical mobile networks is becoming less of an issue as battery technologies are improving and renewable energy sources become available.

Most importantly, the following issues often turn into design objectives or challenges of tactical communications networks [8]:

- Integration and interoperability;

- Scalability and seamless connectivity;

- Mobility and dynamic topologies;

- Security;

- Survivability and reliability;

- Bandwidth-Constrained and variable capacity links;

- Communications support the chain of command.

Our research is motivated by the challenges outlined above. The main motivation for the research presented in this thesis is to improve understanding and review how tactical networks are deployed, managed and operated in realistic challenging environments, for example, due to adverse propagation conditions, lack of supporting infrastructure, spectrum limitations, and the stringent requirements for the service security and safety in the mission-critical scenarios. At the same time, the thesis focus is influenced by the student's involvement in a collaborative research and development (R\&D) project with a defence company.

\section{REFERENCES}

[1] K. Pahlavan and A. H. Levesque, Wireless information networks vol. 93: John Wiley \& Sons, 2005.

[2] M. Series, "IMT Vision-Framework and overall objectives of the future development of IMT for 2020 and beyond," Recommendation ITU, pp. 2083-0, 2015.

[3] C.-K. Toh, Ad hoc mobile wireless networks: protocols and systems vol. 11104: Springer, 2002.

[4] M. Mauve, et al., "A survey on position-based routing in mobile ad hoc networks," IEEE network, vol. 15, pp. 30-39, 2001.

[5] M. McBride and M. Masacioglu, "Control Based Mobile Ad Hoc Networking for Survivable, Dynamic, Mobile Special Operation Force Communications," NAVAL POSTGRADUATE SCHOOL MONTEREY CA2009.

[6] C. Shields, et al., "Fault tolerant mobility planning for rapidly deployable wireless networks," in International Parallel Processing Symposium, 1998, pp. 770-789.

[7] J. Sigholm, "Secure Tactical Communications for Inter-Organizational Collaboration: The Role of Emerging Information and Communications Technology, Privacy Issues, and Cyber Threats on the Digital Battlefield," Högskolan i Skövde (University of Skövde), 2016.

[8] M. Ryan and M. Frater, A tactical communications system for future land warfare: 